\title{
Evolving dendritic morphologies highlight the impact of structured synaptic inputs on neuronal performance
}

\author{
Mohammad Ziyad Kagdi \\ University of Aberdeen, Mental Health, Clinical Research Centre, \\ Cornhill Rd., Aberdeen, Aberdeenshire AB25 2ZH, United Kingdom \\ m.ziyad@kagdi.org
}

\begin{abstract}
Dendrites, the most conspicuous elements of neurons, extensively determine a cell's capacity to recognise synaptic inputs. Investigating its structure and morphological properties unravels the functioning mechanism of neurons that cooperates the process of learning and memory. This research systematically generates a varying topology of dendrites in a multi-compartmental model of a neuron with passive properties and it further explores a cell's integration ability of complex synaptic potentials. The neurons receive an equal number of binary input patterns of synaptic activity and the performance of a cell is gauged by calculating the signal to noise ratio between amplitudes of somatic voltage. The objective is to analyse the types of input pattern in combination with morphological properties that may strengthen or weaken the somatic response. Finally, an evolutionary algorithm produces a fine variety of branching structures calculating the weighted sum of synaptic inputs, further identifying the impact of membrane and morphological properties on neuronal performance.
\end{abstract}

Keywords: Dendritic morphology, Synaptic integration, Synaptic plasticity, Hebbian learning, Pattern recognition, Evolutionary algorithm.

\section{Introduction}

A neuron is a nerve cell excited electrically to process and transmit information through electrochemical signals. Many different types of neurons exist in the human brain - approximately $10^{11}$ to $10^{12}$ in number - with a great variety of morphologies. Each neuron connects on average to $10^{14}$ other neurons, constituting a total of $10^{15}$ to $10^{16}$ connections known as synapses [14], thereby establishing the broader realm of human perception, emotions, thoughts and memories. There are four typical components of a single neuron; dendrites, soma, axon and axon terminals. Dendrites are tree like branching structures, often extended away from soma (cell body) for hundreds of micrometers $(\mu \mathrm{m})$, play an important role in propagating and integrating synaptic potentials [2], [13]. Soma is the neuronal cell body attached to dendrites, containing nucleus and other cellular components, and is responsible for sending and receiving electrochemical signals. 
The development of dendrites is regulated by intrinsic genetic factors and cellular organisation of actin and microtubule cytoskeleton for the formation of pertinent dendrite morphology [10]. Dendrites considerably vary in their anatomical structure and are thought to have associated with variety of computational tasks. Dendritic spine is a microscopic membranous protrusion on neuron's dendrite, containing postsynaptic compartment of excitatory synapse to serve as a storage site for synaptic strength [10]. Transmission of electrical stimulations from other neurons is carried out via microscopic junctions called synapses which are located at the various points (spines) across the dendritic arbor. The primary interest of studying the human brain lies in exploring the information processing mechanism among different types of neuronal morphologies. Neurons show a lot of variability in their shape and structure, exhibit a wide variety of patterns and strength of connections through which memories are stored and habits are learned. There have been many investigations done on neurons with different types and morphologies, yet the reason behind these varieties and functional implications of different morphologies remains unclear. Few studies have hypothesised that the variability in morphological structures is unlikely to be accidental and that these variabilities could exist due to optimised propagation of neuronal signals from synapses to soma [2], [13]. Synaptic integration is a complex process that comprises a great deal of computations within dendrites, and requires concurrent inputs from excitatory synapses to determine neuronal firing behaviour. It is one of the possible roles of neuronal dendritic arborization to recognise structured synaptic inputs through integrating various arriving signals at the soma.

The present study focuses on the development of dendritic arbors using partition notations to mimic its tree-like structure and it further evaluates the impact of different structures on a cell's pattern recognition capacity. The developmental approach used here for dendritic growth is based on two different methods. Firstly, a branching stochastic approach is used with partition notations [12] to generate a possible number of dendritic branches and then, an evolutionary algorithm (EA) is utilised to produce an optimum dendritic structure suitable for recognising synaptic inputs. A neuron receives input signals from many other neurons attached to it and the strength of those signals is defined by its synaptic plasticity. To characterise a neuron based on its passive properties, a compartmental model [7] is utilised to simulate the postsynaptic integration of excitatory inputs. The compartmental model of a neuron divides each complicated dendrite into number of compartments and imitates the behaviour of a biologically realistic neuron. On the contrary, an artificial neural network model (ANN) of an associative memory, implementing the Hebbian learning rule is also employed to calculate the weighted sum of its synaptic inputs, thereby comparing its pattern recognition performance with that of the compartmental model neuron. To optimise a neuron's information processing capability, some binary patterns called stored patterns, representing synaptic inputs are presented during a learning phase. Once the learning phase is completed, some novel binary patterns are presented to discriminate them from the stored patterns. A neuron's discrim- 
inatory ability is dependent upon its dendritic structure, spatially distributed excitatory inputs and strength of connections as measured by the signal to noise ratio $(\mathrm{S} / \mathrm{N})$ between the somatic EPSP amplitudes / weighted sum responses of the stored and novel patterns. The higher the $\mathrm{S} / \mathrm{N}$ ratio, the better able the cell to discriminate the two sets of input patterns. More importantly, it is also hypothesised that the spatial distributions of synaptic inputs could affect the postsynaptic response, as a result, it may strengthen or weaken the neuronal performance by sending signals to the soma from shorter or longer dendritic distance. To identify whether this is correct, current study also focuses on presenting some biased synaptic input patterns called fixed stored patterns in which input locations on dendrite are manually determined. Additional parameters such as axial resistivity, compartment and mean path length, temporal asynchrony of signal arrival are also investigated to ascertain whether these parameters have any association with the cell's pattern recognition performance.

\section{The Model}

The primary aim of this study is to understand the functioning mechanism of a neuron that cooperates the process of learning and memory by storing and recognising synaptic inputs. To begin with, neuron models are presented with membrane and morphological properties (Section 2.1 and 2.2), after which an EvOL-DnDR ${ }^{1}$ algorithm generates a population of 100 neuron models with diversified dendritic topologies, which are further provided with the sets of synaptic inputs and their performances are measured by calculating the $\mathrm{S} / \mathrm{N}$ ratio.

\subsection{Passive membrane properties}

A passive neuron model is used to understand its electrical properties without any active conductances in the soma and dendrites, therefore it does not generate action potentials. From the study carried out by De Sousa [3], the following passive parameters are considered for membrane capacitance, membrane resistance and axial resistivity, respectively: $C_{\mathrm{m}}=0.75 \mu \mathrm{F} / \mathrm{cm}^{2}, R_{\mathrm{m}}=30 \mathrm{k} \Omega \mathrm{cm}^{2}$ and $R_{\mathrm{a}}=150 \Omega \mathrm{cm}$.

\subsection{The compartmental model of a neuron}

In the compartmental model, a neuron is treated as a cell body with divided isopotential dendritic compartments, receiving input signals in the middle of every compartment as shown in Fig. 1 (C). The charge across each compartment is same and can be represented by an electrical circuit. The length and diameter of soma is based on the study carried out by De Sousa [3] where the soma is a cylinder of $20 \mu \mathrm{m}$ length and diameter, followed by $500 \mu \mathrm{m}$ length and 25 $\mu \mathrm{m}$ diameter for each dendritic compartment. The conductance amplitude of a

\footnotetext{
${ }^{1}$ http://research.kagdi.org/cns/evolving-dendritic-morphologies
} 
naive synapse is set to $1.5 \eta \mathrm{S}$ (before learning) and is subject to change once multiplied with the weight value after synaptic learning. The resting membrane potential of a model neuron was set to $-65 \mathrm{mV}$ before the transmission of any excitatory inputs. On arrival of these input signals, an excitatory postsynaptic potential (EPSP) amplitude, representing the depolarisation of membrane voltage is generated. An EPSP is the somatic excitation needed for any neuron to fire an action potential (AP), caused by the incoming active signals.

\subsection{Generation of dendritic structures}

To define the growth of a multi-compartmental dendrite, partition notations are used involving axioms and rules to constitute the branching structure [12]. In a binary tree, a partition at the bifurcation point is defined by a pair of numbers denoting the degree of each subtree, where the terminal nodes in each subtree are further divided into left and right branches as tree grows. Therefore, a sequential specification containing partition notations executes in a linear order to bifurcate and generate branches of a tree. For instance, a tree with 4 terminal nodes and 7 compartments can be specified using the partition notations $4(2(1,1) 2(1,1))$

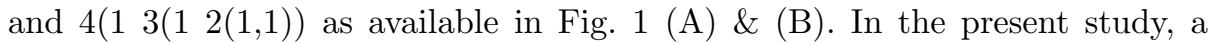

(A)

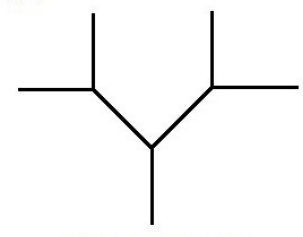

$4(2(1,1) 2(1,1))$
(B)

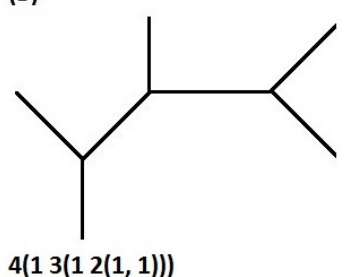

(C)

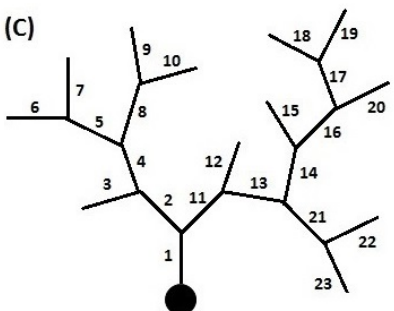

Fig. 1. Partition notations used to define dendritic trees with 3 terminal nodes and 7 compartments in (A) \& (B), and Branching of a neuron using the compartmental model in (C).

population of 100 neurons is generated with $\mathrm{T}=128$ terminal nodes and 255 $(=2 \mathrm{~T}-1)$ compartments, introducing different types of dendritic topologies by stochastically determining number of terminal points at every branch in a tree.

\subsection{Synaptic plasticity and long-term potentiation}

A learning process in neurons demonstrates the cells' activity-dependent adaptive behaviour characterised by the Hebbian learning rule of growing synaptic strength between the firing neuron and the neuron receiving input signals called synaptic plasticity [6]. A change in synaptic strength occurs due to persistent stimulations by pairs of pre and post synaptic neurons, where a persistent increase in synaptic strength represents Long-term potentiation (LTP) [11]. LTP 
is an input-specific process shaped by pre-synaptic activations which as a result determines the behaviour of a postsynaptic neuron. To achieve the synaptic strength in simulated neurons, a weight value is used that increases by 1 , every time there is an active synapse established between the pre and post synaptic neurons, which consequently stores a history of active connections representing neuronal activities, as shown in Fig. 2 (A).

\subsection{The pattern recognition task}

The ability of a neuron to discriminate between patterns of synaptic inputs is largely dependent upon its ability to recognise number of active synapses [4]. Comparison is made between the performance of a computing unit in an artificial neural network (ANN) model of an associative memory and the compartmental model of a biologically realistic neuron. At the outset, two sets of 10 binary synaptic input patterns, called the stored and the novel patterns are provided to the computing unit in ANN. Each pattern contains 255 bit series of randomly generated binary values representing active and inactive synapses (where 1 denotes an active synapse) presented to each unit's 255 input layers. There are 25 active synapses in each pattern. Under the training phase, the unit learns 10 stored patterns, and it discriminates the 10 stored patterns from the 10 novel patterns when tested in the recall phase. On each active synapse from the

\section{(A) Learning}

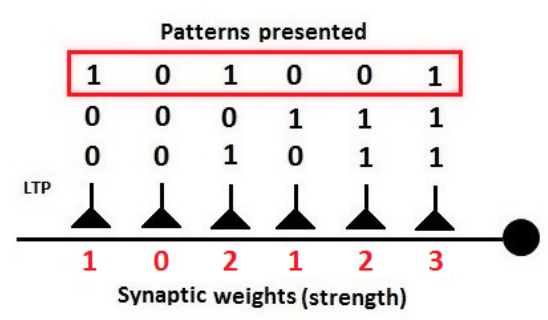

\section{(B) Recall}
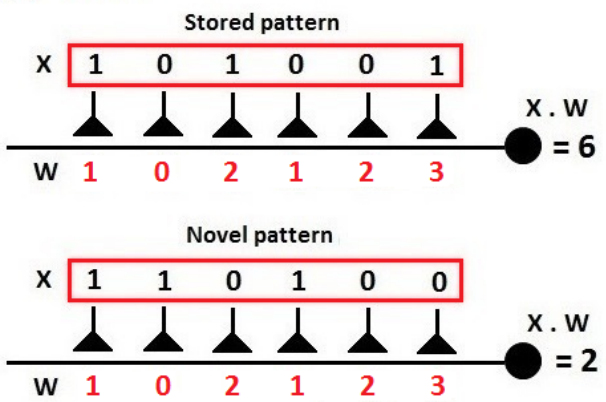

Fig. 2. LTP learning with 3 synaptic input patterns in (A), and a Recall phase with synaptic weights to recognise patterns in (B).

stored patterns, a typical weight value is increased by 1 , describing the concept of synaptic strength, as shown in Fig. 2 (A). A response to a specific pattern is a sum of all inputs multiplied by the associated synaptic weights (Dot product of an input and weight value) representing the dendritic summation, as calculated for both the pattern sets using Eq. 1 and is shown in Fig. 2 (B). The dendritic sum of an output unit is considered as an ultimate response to each pattern presented, and it is used to calculate the $\mathrm{S} / \mathrm{N}$ ratio using Eq. 2 [3]. 
In the secondary aspect of the pattern recognition task, both sets of input patterns along with the associated synaptic weights are transferred to the NEURON compartmental model [7]. In a population of 100 compartmental model neurons with varying dendritic morphologies, the 255 binary inputs are spatially distributed to each neuron's 255 dendritic compartments (synapses), each active input arrives synchronously at its associated synaptic location. The resultant EPSP amplitudes of each compartmental model neuron for both the pattern sets were recorded. The EPSP amplitudes represent a change in each cell's somatic voltage, showing the higher and lower membrane potentials, are considered as a response to the sets of input patterns provided. There is a strong association between the somatic EPSP amplitudes and a cell's possible AP [4], indicating that the $\operatorname{EPSP}(\mathrm{s})$ play a crucial role in determining the cell's pattern recognition performance. The generated EPSP amplitudes were used to calculate the S/N

\section{(A) EPSP Responses}

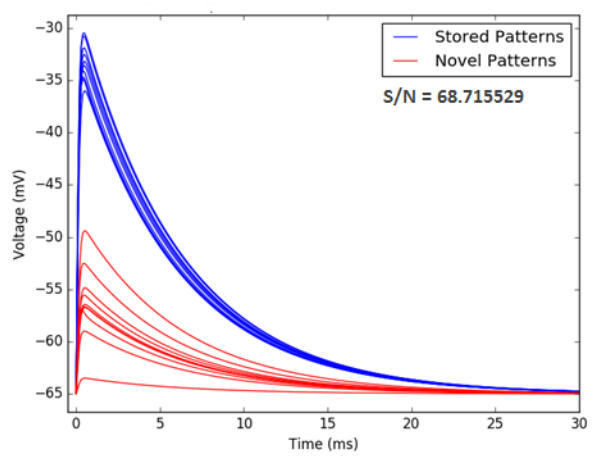

\section{(B) Frequency of the Peak Responses}

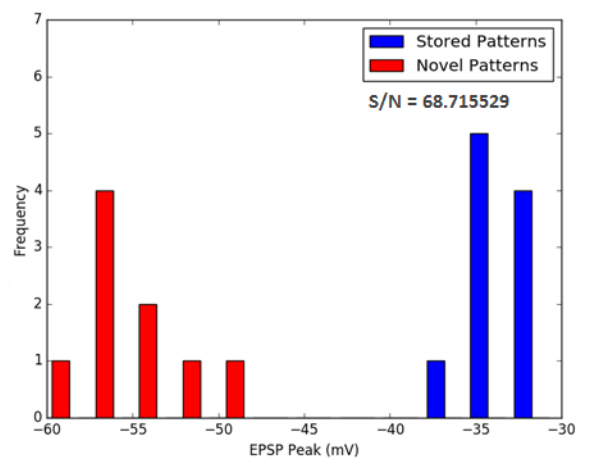

Fig. 3. The EPSP amplitudes of passive neuron to 10 stored and 10 novel patterns in (A), and the frequency of the peak EPSP responses to both stored and novel patterns (bin width $=1 \mathrm{mV}$ ) in $(\mathrm{B})$.

ratio between the responses of stored and novel patterns, which later compared with the performance of a computing unit in an ANN model. Various forms of noise that degrade spatio-temporal integration of synaptic inputs are absent in an ANN model, which are investigated in the compartmental model neurons. A high performing neuron was thus identified as the one with maximum $\mathrm{S} / \mathrm{N}$ ratio in a population of 100 compartmental model neurons, discriminating between the sets of synaptic inputs, as shown in Fig. 3 (A). The following equations are used to calculate the Dendritic Sum and the S/N ratio.

$$
\text { Dendritic Sum }=\sum_{i=1}^{n} X_{i} . W_{i} .
$$

In Eq. 1, $n$ refers to the number of synaptic compartments / weights which is 255 for each binary pattern, $X_{i}$ is the $i^{\text {th }}$ input signal, $W_{i}$ is the $i^{\text {th }}$ weight value 
in the associated synaptic pattern, as shown in Fig. 2 (A) and (B).

$$
S / N=\frac{\left(\mu_{s}-\mu_{n}\right)^{2}}{0.5\left(\sigma_{s}^{2}+\sigma_{n}^{2}\right)} .
$$

In Eq. $2, \mu_{s}$ and $\mu_{n}$ are the mean values and $\sigma_{s}^{2}$ and $\sigma_{n}^{2}$ are the variances of the ultimate / peak responses of stored and novel patterns. Graham proposed a similar type of LTP learning model, calculating the $\mathrm{S} / \mathrm{N}$ ratio and discriminating the synaptic inputs in the presence and absence of noise in a multi-compartmental model of a CA1 hippocampal pyramidal neuron. A study which concluded that the amplitudes of voltage responses at the soma is dependent upon the spatial distribution of synapses and that the variations in amplitudes occur due to different synaptic locations (which as a result cause the temporal asynchrony of signal arrival at the soma) even with the same number of active synapses [4].

\subsection{Mean path length}

The mean path length is the measure of dendritic distance from soma to terminal points, considered to analyse the performance of a neuron. Eq. $3 \& 4$ denote the dendritic and synaptic mean path (DMP and SMP) length, calculating the average sum of path lengths (mean number of compartments) from soma to terminal points, and the average sum of path lengths from soma to number of active synapses respectively.

$$
\begin{aligned}
& \text { Dendritic Mean Path }=\frac{1}{n} \sum_{k=1}^{n} P_{k} . \\
& \text { Synaptic Mean Path }=\frac{1}{n} \sum_{k=1}^{n} Q_{k} .
\end{aligned}
$$

Here, $P_{k}$ is the length of dendritic path to the $k^{t h}$ terminal point, and $Q_{k}$ is the length of dendritic path to the $k^{t h}$ synaptic compartment from the soma. Fig. 4 shows the calculated DMP and SMP length for each tree available, where a red line represents an active synapse to that particular compartment.
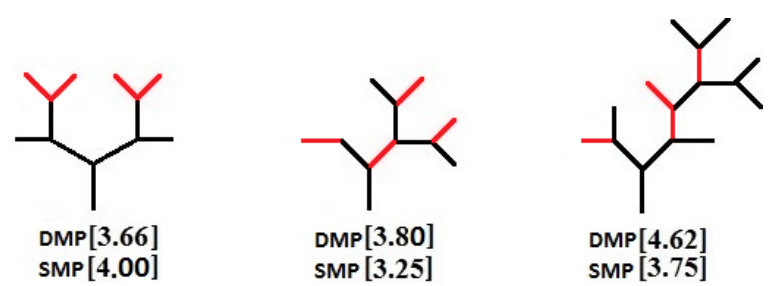

Fig. 4. Dendritic and synaptic mean path (DMP and $S M P)$ lengths are calculated. 


\subsection{Evolutionary algorithm and exploration of dendritic structures}

An evolutionary algorithm (EA) is a heuristic optimisation technique inspired by the Darwinian theory of survival of the fittest and natural genetics, applied to a population of individuals for breeding high quality solutions [9]. In the present study, an EA is implemented with a population of 100 candidate solutions (compartmental neurons) to produce a fine variety of branching structures and to maximise the $\mathrm{S} / \mathrm{N}$ ratio. It is the secondary approach of producing some functionally desirable dendrites in each generation by only selecting high performing neurons (with maximum $\mathrm{S} / \mathrm{N}$ ratio) for reproduction. Once pairs of individuals are selected, the genetic features of dendrites are exchanged using dual point crossover and offspring are mutated with $4 \%$ to $20 \%$ of a mutation rate. Additionally, the concept of elitism is also utilised, keeping about $10 \%$ to $15 \%$ of best individuals intact in the next generation without any genetic modifications. Elitism makes sure that the EA does not lose high performing neurons once their genetic details are exchanged or mutated to produce future offspring. The above steps are repeated until some most fit neurons are reproduced.

\section{$3 \quad$ Results}

\subsection{Distortion of input signals in dendritic trees}

Membrane potential plays a crucial role in determining neuronal performance, and therefore it is essential to identify the causal relationship between the travelling inputs and the attenuation of an EPSP response. On a dendritic tree, individual synapses may differ in their distance from soma. As a result, the amplitude of voltage response at the soma can be affected by the spatial distribution of these input signals as they travel along the lengthy dendritic tree to the final integration site. Fig. 5 shows examples of voltage responses generated at the soma due to different distribution of synaptic inputs (blue traces) on the dendritic tree, whereas variations in voltage amplitude occur, such as an EPSP amplitude decreases and its time span lengthens, with the distance of an originating synapse from the soma. An EPSP response of 22 sparsely attached active inputs to the 22 distal dendritic compartments in Fig. 5 (A) \& (B) shows only about $0.5 \mathrm{mV}$ of postsynaptic depolarisation as opposed to $8.5 \mathrm{mV}$ when inputs are clustered proximally to the soma, see Fig. 5 (C) \& (D). Interestingly enough, despite that the distributed excitatory inputs arrive synchronously at individual synapses, the spatio-temporal integration of transient signals is still disrupted due to asynchronous arrival of excitatory potentials at the soma, significantly affected by the varying synaptic distances from the cell body. Fig. 5 (B) \& (D) shows calculated temporal asynchrony of signal integration and the synaptic mean path length (SMP) depicting larger value for distorted and delayed membrane voltage. Similarly, the higher $R_{\mathrm{a}}$ of individual compartment increases the intracellular resistance for ions to move and disrupts the flow of synaptic potentials. Hence, the amount of signal attenuation witnessed here is proportional to the value of axial resistivity used $\left(R_{\mathrm{a}}=120 \Omega \mathrm{cm}\right)$, showing inverse association with degrading membrane potential. 
(A)

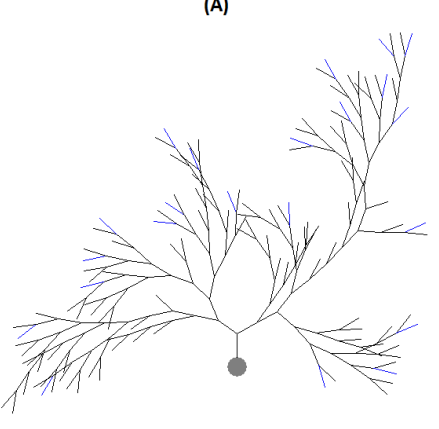

(c)

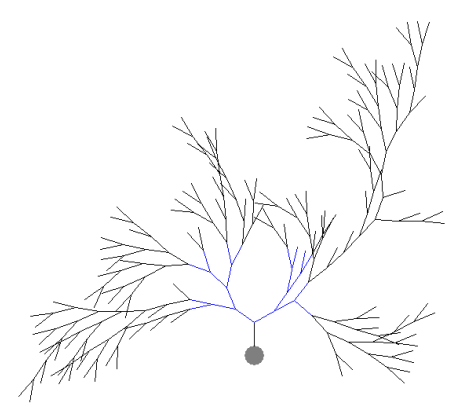

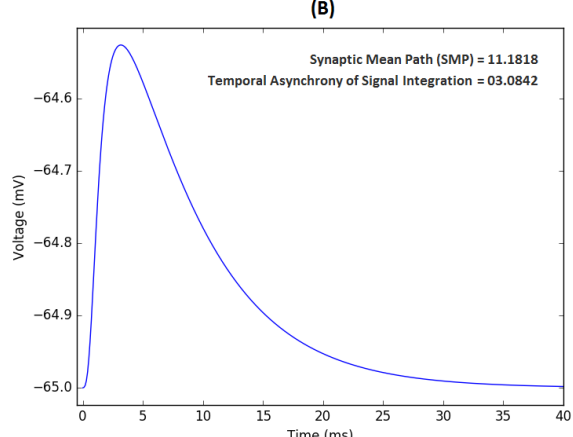

(D)

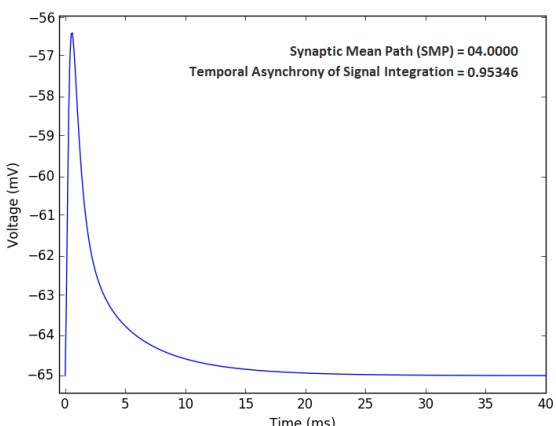

Fig. 5. 22 active synaptic inputs are attached to compartments at distal / proximal to the soma in (A) \& (C) and their voltage responses in (B) \& (D) with calculated Synaptic Mean Path (SMP) and Temporal Asynchrony of Signal Integration.

\subsection{Discrimination of synaptic inputs and the signal to noise ratio}

Before measuring the somatic EPSP responses of a compartmental model neuron, two sets of randomly generated synaptic input patterns were provided to the computing unit in an ANN model and their dendtric sums were calculated using Eq. 1, see frequency distribution in Fig. 6 (A). The s/n ratio was 40.1993 generated from the dendritic sums of both the pattern sets. Although, the distinction is quite clear, it is important to measure the somatic response in the compartmental model neuron. Once measured, the resultant $\mathrm{S} / \mathrm{N}$ ratio was 10.130346 with some rather overlapping EPSP amplitudes (results not shown). The DMP and SMP lengths were 9.531 and 8.448, calculated using Eq. 3 \& 4 respectively. Due to the wider distribution of input synapses, the $\mathrm{S} / \mathrm{N}$ ratio was relatively lower, showing poor discrimination of stored and novel patterns. To produce some high performing neurons, an EA was utilised, evolving 100 individuals for 100 number of generations, which finally produced an optimised neuron (Appendix Fig. 1 A) showing discriminatory EPSP amplitudes with its improved $\mathrm{S} / \mathrm{N}$ ratio of 48.987556 , as shown in Fig. 6 (C) \& (E). Performance of a most fit neuron in each generation was compared against its SMP and DMP length which showed a negative correlation of -0.7937 and -0.4947 respectively, indicat- 
ing growing improvements in the neuronal performance $(\mathrm{S} / \mathrm{N}$ ratio) with closer synapses to the soma, see Fig. 7 (A) \& (B). Whilst this is the case, it is also key
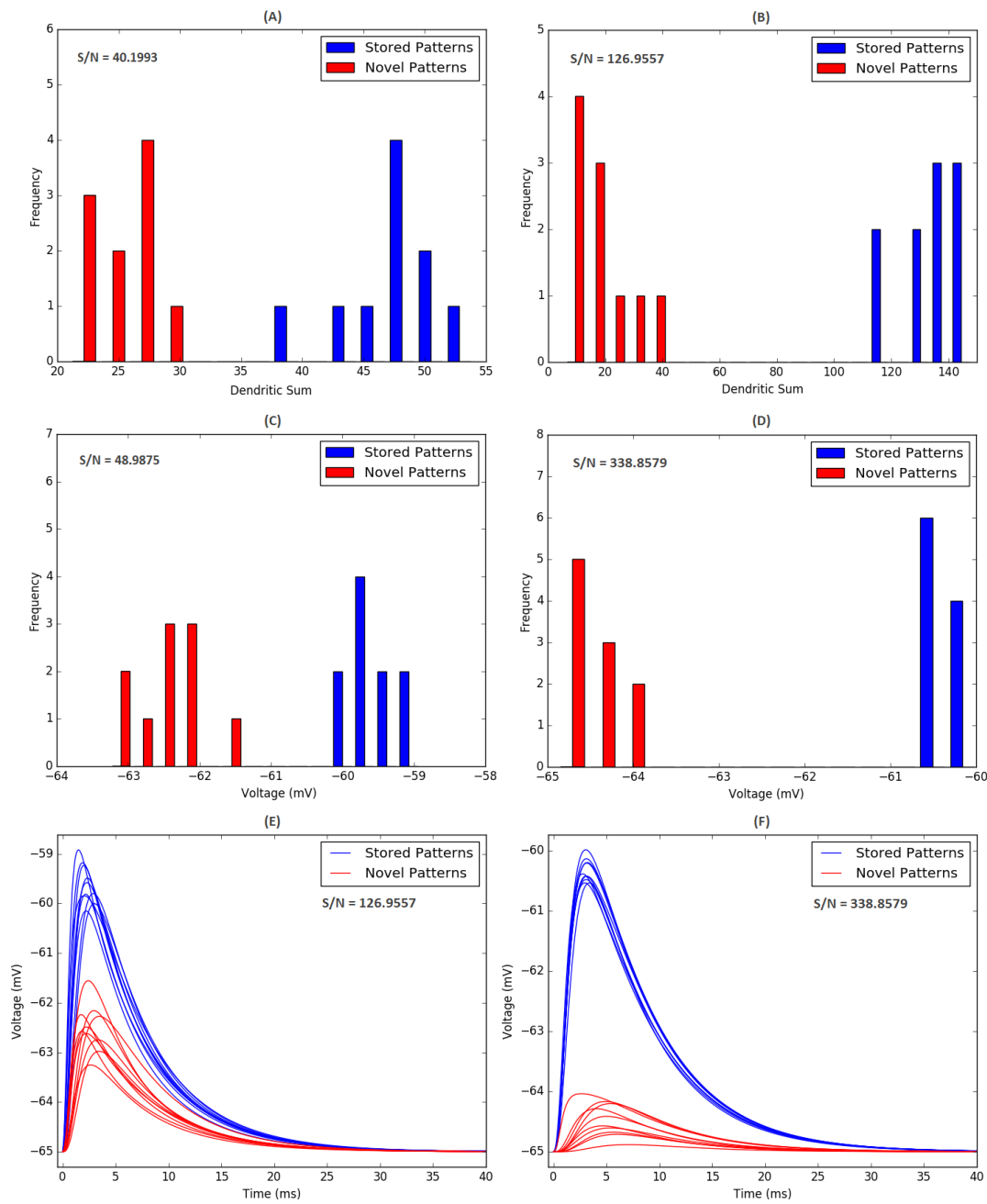

Fig. 6. Evolution of dendrites for recognising random and clustered (fixed) synaptic inputs, showing frequency distribution of an ANN model's weighted sums in (A) \& (B), frequency distribution of an evolved neuron's peak responses in (C) \& (D), and their associated EPSP amplitudes in (E) \& (F) respectively.

to measure the significance of clustered synapses, when input signals are manually arranged. Synaptic strengthening of a cell in forms of weight values depends however on where, how many and how often synapses are activated. A set containing 10 fixed stored patterns was designed by variably concentrating $20 \%$ to $25 \%$ of active inputs at 4 different regions across the dendritic tree. These spa- 
tial arrangements of synaptic inputs played a major role for somatic integration by controlling the strength of combined active signals. The calculated neuronal performance was 126.9557 in an ANN model, frequency distribution is shown in Fig. $6(\mathrm{~B})$. On the contrary, the maximum $\mathrm{S} / \mathrm{N}$ ratio of 83.5114 was found (results not shown) in a population with 100 compartmental neurons, which further increased to 338.8579 after 100 evolutionary iterations (the evolved neuron is in Appendix Fig. 1 B), showing a clear distinction between the synaptic patterns, as indicated in Fig. 6 (D) \& (F). Again, the $\mathrm{S} / \mathrm{N}$ ratio for each generation was compared against the SMP and DMP lengths, and a negative association of -0.4210 and -0.3258 was recorded as shown in Fig. 7 (C) \& (D) respectively.
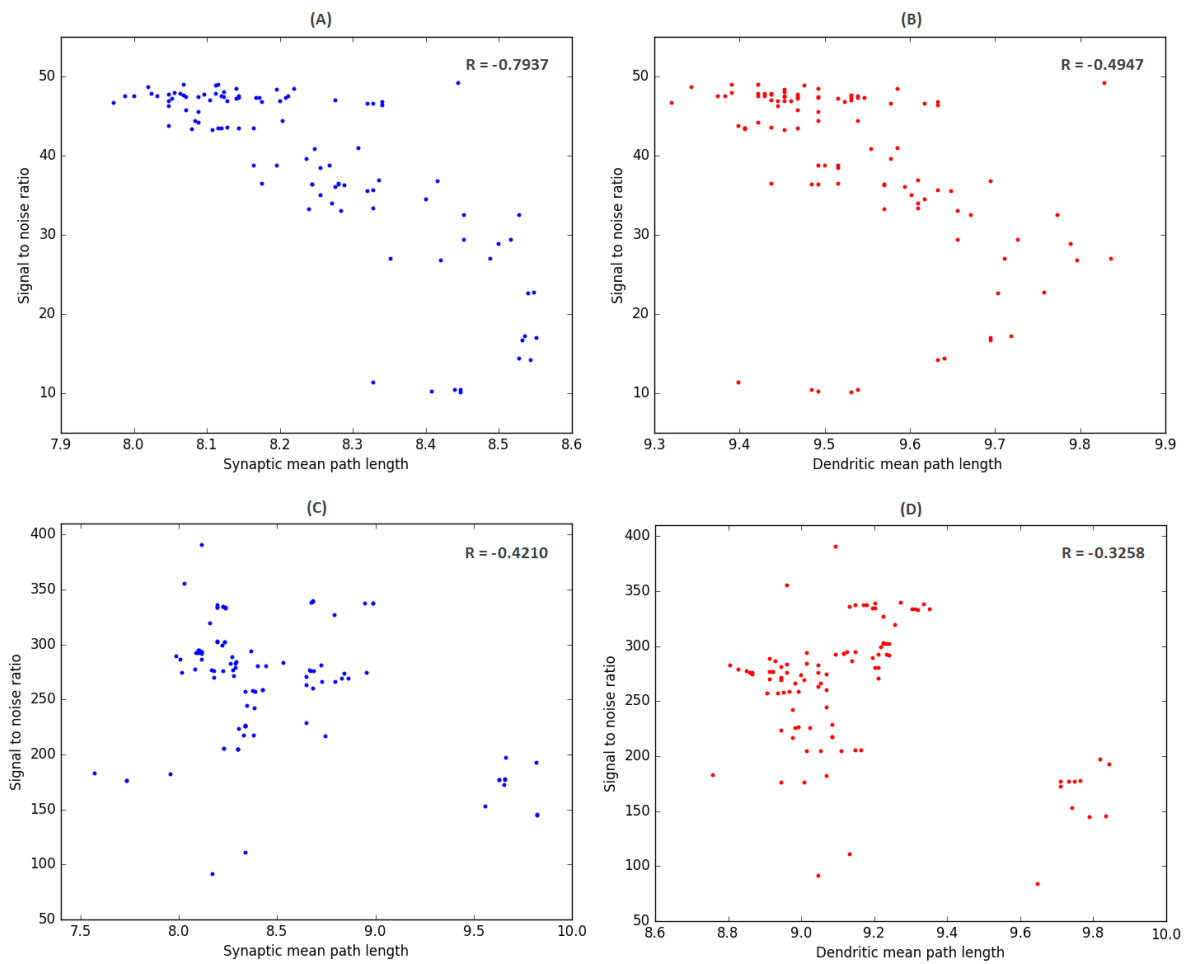

Fig. 7. The $\mathrm{S} / \mathrm{N}$ ratio of a most fit neuron from each evolutionary generation was compared against its SMP and DMP length, recognising both the random and clustered input patterns in (A), (B) \& (C), (D) respectively.

\subsection{Temporal asynchrony of somatic integration}

Since the random and a wide distribution of synaptic inputs show a great variety of distances from soma, there exists a temporal asynchrony of signal arrival at the final integration site. An effect of which broadly affects the neuronal response by making it highly inhomogeneous, as shown in Fig 5. Therefore, it is 
also likely that the variety of these EPSP responses are proportional to the temporal irregularity of incoming inputs. It would be useful to ascertain whether these temporal irregularities of signal arrival have a causal relationship with their associated inhomogeneous somatic responses. An experiment was carried out in which an EA recorded the temporal irregularity of signal arrival for each most fit neuron for 100 generations. Once they were compared with the varying peak responses of stored patterns, a positive association of 0.631279039 was found, suggesting growing variations of somatic responses with increasing temporal asynchrony of signal arrival at soma. Similarly, the temporal asynchrony was also compared against the measure of $\mathrm{s} / \mathrm{n}$ ratio which depicted a negative correlation of -0.5151 , showing poor neuronal performance with a growing synaptic irregularity, see Fig. 8.

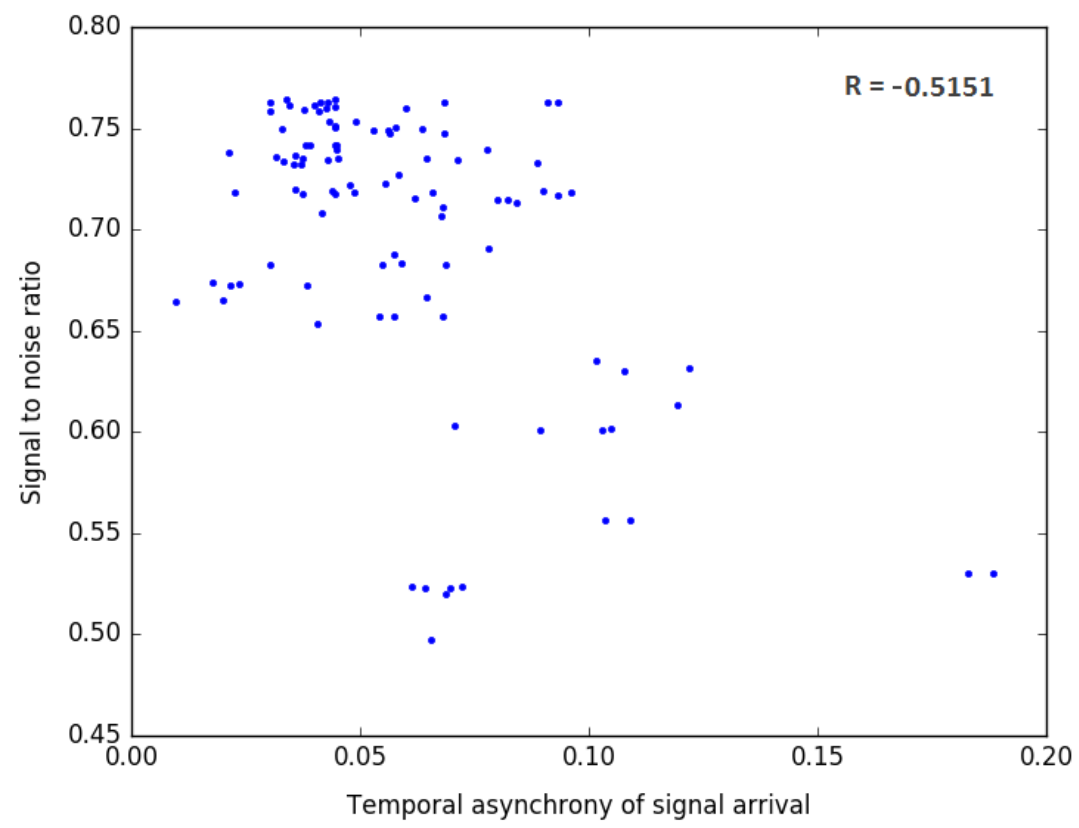

Fig. 8. Temporal irregularity of signal arrival at soma and its impact on the $\mathrm{S} / \mathrm{N}$ ratio.

\section{Discussion}

The pattern recognition capacity of a cell was assessed in an artificial neural network model of an associative memory, discerning between two sets of transient input patterns. To estimate the likely effect of different dendritic topologies and the impact of spatio-temporal noise on neuronal performance, a compartmental model simulated the postsynaptic integration of synaptic inputs. The performance of a neuron is directly associated with the spatial distribution of its 
synapses. Consequently, it performs sufficiently well when inputs are clustered as opposed to when they are purely random. Persistent and clustered synaptic inputs to a specific region of a dendrite enhances the strength and arrival timing of these signals at soma, complying with the Hebbian learning rule for long-term potentiation and synaptic plasticity. However, when inputs are randomly generated, a neuron receives these signals widely across its dendritic arbour, attaining apportioned synaptic weights to exhibit its average discriminatory performance. The EA successfully generated best possible individuals in each generations and the evolved neurons performed sufficiently well compared to the originally generated neurons. Considering the impact of different dendritic distance on neuronal performance, a measure of mean path length was compared with the $\mathrm{S} / \mathrm{N}$ ratio of each evolving neuron from the population. An outcome of this showed a negative association of mean path lengths with improving neuronal performance. Furthermore, the obvious impact of temporal irregularity introduced due to different synaptic distances from soma, as explained in section 3.3, showed a growing association with the variability of somatic voltage amplitudes. Whilst there is a relationship between the two variables, it is still least relevant to support the assertion that such irregularity causes other variations to occur. It can be argued due to the fact that attenuation or strengthening of somatic voltage does not solely dependent upon such temporal irregularities, and that the arrival of such irregular inputs could still perform better if they are clustered and if arrived with strong potentials, as it was the case with fixed synaptic patterns (Section 3.2 ), suggesting weak relevance with the dependency of synaptic distance.

The measure of $\mathrm{S} / \mathrm{N}$ is proportional to the differing responses between the stored and novel patterns, where the reduction in variability of each of the pattern set's peak responses directly influence the cell's pattern recognition performance. Performance of a neuron with a large number of active synapses (in stored patterns) nearer to soma depicts a least variability of somatic voltage amplitudes, since there is a smaller variance of synaptic distances. As a result, arriving signals get integrated at soma more or less synchronously, also enhancing the measure of signal to noise ratio.

The increasing value of compartment length and axial resistivity, $R_{\mathrm{a}}$ exhibit a degrading and prolonged amplitude of somatic voltage. To verify these effects, different values such as 200, 300, $400 \mu \mathrm{m}$ for compartment length and 50, 100, $150 \Omega \mathrm{cm}$ for $R_{\mathrm{a}}$ were considered, which showed decreasing mean stored $\left(\mu_{s}\right)$ responses; -57.02, -59.79, -61.20 (each affected by an increasing compartment length) and $-57.70,-59.35,-60.31$ (each affected by an increasing $R_{\mathrm{a}}$ ) respectively. An appropriate value of axial resistivity is quite uncertain and it was estimated between 50 - $400 \Omega \mathrm{cm}$ [4]. A relatively lower value of $R_{\mathrm{a}}$ improves the $\mathrm{S} / \mathrm{N}$ ratio by decreasing intracellular resistance for ions to move, ultimately reducing the variability of somatic responses to stored and novel patterns.

The present study is an extension of work done by Graham [4] and De Sousa [3] in which synaptic patterns were composed of synchronously arriving randomly generated transient inputs. A study carried out by Graham [4] involved pattern recognition of 100 and 200 active inputs, which in the current 
study are considered as stored and novel patterns, but with equal number of active inputs. Currently, a notion of clustered synaptic inputs is used in addition to the randomly generated input patterns along with an EA to optimise number of inputs that each active synapse receives. Clustered synapses aim to minimise the distance variations of inputs to soma and grow the membrane voltage in each cluster by controlling the strength of combined signals - which as a result produce least variable EPSP amplitudes for an improved S/N ratio. The implication of this findings is that the spatial organisation of active inputs determines dendritic integration of a postsynaptic neuron with a little dependency on any specific structure of a morphology. In other words, a correlated and synchronous arrival of inputs to soma is the best predictor for high performing neurons.

Acknowledgements. I would like to express my sincere gratitude to Dr Rene te Boekhorst for his valued support and guidance extended to me.

\section{References}

1. Cooke, S. F., and T. V. P. Bliss.: Plasticity in the human central nervous system. Brain, 129.7, 1659-1673 (2006)

2. Cuntz, Hermann, Alexander Borst, and Idan Segev.: Optimization principles of dendritic structure. Theoretical Biology and Medical Modelling, 4.1, 1 (2007)

3. De Sousa, Giseli, et al.: Dendritic morphology predicts pattern recognition performance in multi-compartmental model neurons with and without active conductances. Journal of computational neuroscience, 38.2, 221-234 (2015)

4. Graham, Bruce P.: Pattern recognition in a compartmental model of a CA1 pyramidal neuron. Network: Computation in Neural Systems, 12.4, 473-492 (2001)

5. Gulledge, Allan T., Bjrn M. Kampa, and Greg J. Stuart.: Synaptic integration in dendritic trees. Journal of neurobiology 64.1, 75-90 (2005)

6. Hebb, Donald O.: The organization of behavior: A neuropsychological theory. Psychology Press (2005)

7. Hines, Michael L., and Nicholas T. Carnevale.: The NEURON simulation environment. Neural computation, 9.6, 1179-1209 (1997)

8. Ho, Victoria M., Ji-Ann Lee, and Kelsey C. Martin.: The cell biology of synaptic plasticity. Science, 334.6056, 623-628 (2011)

9. Langton, Christopher G.: Artificial life: An overview. Mit Press (1997)

10. MartnezCerdeo, Vernica.: Dendrite and spine modifications in autism and related neurodevelopmental disorders in patients and animal models. Developmental Neurobiology (2016)

11. Takeuchi, Tomonori, Adrian J. Duszkiewicz, and Richard GM Morris.: The synaptic plasticity and memory hypothesis: encoding, storage and persistence. Phil. Trans. R. Soc. B, 20130288, 369.1633 (2014)

12. Van Pelt, J., and R. W. H. Verwer.: Growth models (including terminal and segmental branching) for topological binary trees. Bulletin of mathematical biology, 47.3, 323-336 (1985)

13. Wen, Quan, and Dmitri B. Chklovskii.: A costbenefit analysis of neuronal morphology. Journal of neurophysiology, 99.5, 2320-2328 (2008)

14. Williams, Robert W., and Karl Herrup.: The control of neuron number. Annual review of neuroscience, 11.1, 423-453 (1988) 


\section{Appendix:}
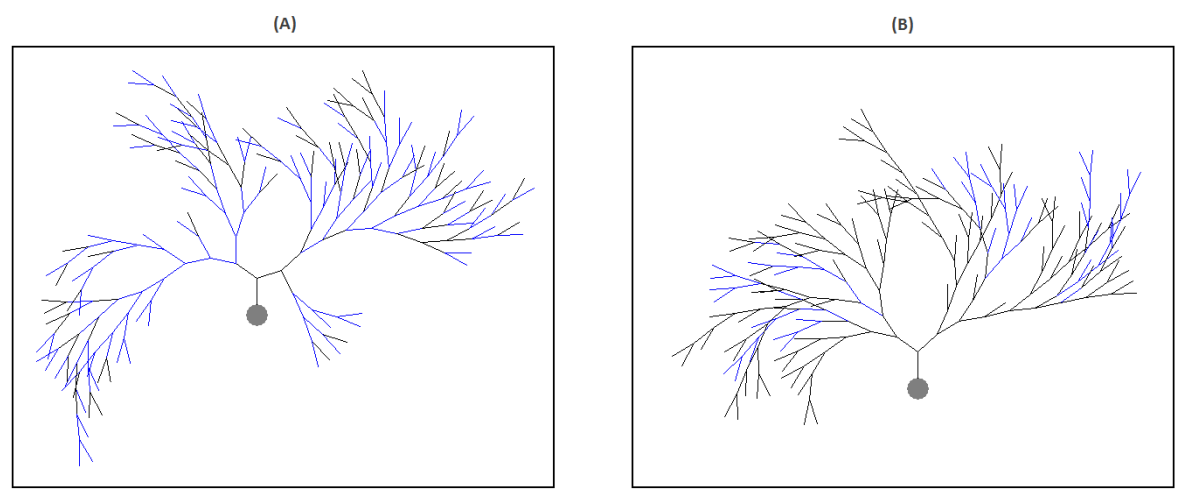

Fig. 1. Dendritic morphologies evolved after 100 evolutionary iterations to recognise random and clustered input patterns in (A) \& (B) respectively. 